\title{
Les aspects et les enjeux du silence dans Jacques le fataliste de Diderot
}

Dans Jacques le fataliste, Diderot raconte l'histoire de deux voyageurs, Jacques et son Maître, qui entreprennent un parcours vers une destination indéterminée. Sur leur chemin, ils ne cessent de parler. Tantôt ils se débattent sur des sujets philosophiques, tantôt ils se racontent des souvenirs d'enfance et des anecdotes. Diderot intervient fréquemment : il coupe la parole aux deux protagonistes pour introduire soit ses commentaires sur ce qu'ils disent, soit pour dire ce qu'il leur pourrait faire dire ou subir, soit pour introduire des réflexions à travers des récits anecdotiques. Les interruptions ne concernent pas seulement le récit initial. Elles ponctuent également les récits enchâssés entrepris par Jacques, par son Maître et par d’autres personnages, telle l'hôtesse. Le roman présente ainsi une véritable fresque où s'interpénètrent récits d'amours, récits d'aventures, scènes théâtralisées, débats philosophiques, réflexions littéraires, portraits, etc. Toutefois la prolixité des séquences discursives et narratives ${ }^{1}$ laisse voir des " non-dits » et des " manques ». Les interlocuteurs, en fait, taisent souvent une information, un épisode ou une émotion. Leur silence revient comme un leitmotiv. Il crée le vide sans pour autant ne « rien » dire (Van Den Heuvel, 1985 : 68). Quels sont donc les différents aspects du silence sous la plume de Diderot et quels en sont les enjeux?

\section{Les interruptions : des silences et des digressions}

Tous les récits - on en distingue vingt-trois - dans le roman sont des textes discontinus, ponctués de haltes à commencer par le récit initial qui sétend sur neuf

Salwa Ben Sassi-Taktak - docteur, maître-assistante à la Faculté des Lettres et des Sciences Humaines de Sfax, Tunisie. Adresse pour correspondance : BP 86. Code postale 3049 Sfax UMA. Tunisie; e-mail : salwataktak@yahoo.fr

1. Diderot a loué, dans L'Éloge de Richardson, paru en 1762, «la variété des événements et le grand nombre des personnages chez Richardson »(Coulet, $1967: 497)$. 
jours. La narration est coupée par les interventions de Diderot ${ }^{2}$ et par les séquences dialoguées. Celles-ci proposent des débats sur différents sujets mais elles reviennent souvent sur celui du fatalisme. Les personnages, Jacques, son maître, l'hôtesse, le marquis des Arcis, etc. s'engagent dans des récits. Ces orateurs sont régulièrement interrompus par les remarques et les commentaires de leurs auditeurs et du narrateur fictif qui rappelle le retour au récit initial et, bien évidemment, par les commentaires de Diderot qui se complaît à pérorer en fonction du contexte énonciatif. Il parle tantôt d'un poète ("Pondichéry » $(2013: 44)$ ), tantôt d'un philosophe («Socrate » (2013: 82)), ou encore d'un dramaturge («Ésope » (2013:55)). Il peint des portraits (« l'hôtesse « (2013 : 131) /la voisine de Desglands (2013: 274- 275) / l'aubergiste $78 /$ le vicaire $(2013: 233)$ etc.) Il contredit le fatalisme de Jacques (2013: 191-193). Il expose sa manière de raconter et sa relation avec « la dive bouteille» $(2013: 239-241)$. Il commente le caractère d'un personnage (« Mme de La Pommeraye » (2013 : 172174)). Il s'autocritique (2013:236-238). Et bien souvent il interpelle le lecteur en vue de lui rappeler les principes de la création romanesque de son époque qu'il réfute, annonçant aussi qu'il s'en démarque ${ }^{3}$.

Ces interruptions représentent des pauses dans l'énonciation. Leurs occurrences abondent. Elles sont parfois brèves. Parfois elles se prolongent sur des pages. L'orateur rompt son discours narratif, non pour se taire définitivement, mais plutôt pour se permettre de digresser sur un sujet relatif - ou non - au récit qu'il raconte. Ce silence provisoire affecte donc le cours des histoires véhiculées par la narration ; il ne trouble pas l'unité dramatique mais il déjoue régulièrement les attentes du lecteur. Celui-ci en attendant la suite de la narration se trouve emballé soit dans d'autres lectures sur les positions philosophique et/ou littéraire de Diderot soit dans d'autres récits. Cette forme de silence a la particularité de créer le suspense puisqu'elle réalise les atermoiements : le récit de Gousse est raconté sur trois étapes ; l'histoire de Mme de La Pommeraye et du marquis d'Arcis ne s'achève qu'au bout de multiples interruptions ; les amours du maitre subissent également bien des coupures ; celles de Jacques s'étendent sur une multitude d'épisodes, etc.

Les haltes au niveau du récit initial ainsi qu'au niveau des récits enchâssés dans le roman de Jacques le fataliste ne sont pas dues à une «insuffisance du langage [ou à une] aphasie " (Van Den Heuvel, 1985 : 67). Elles s'investissent d'une fonction pragmatique : elles écrivent le silence. Cette forme de silence autorise la digression, favorise l'éclatement des idées, permet de briser l'illusion romanesque et cautionne la condensation. Diderot s'en sert sciemment. Il semble privilégier une attention particulière au dialogue et à la dialectique des idées. L’emploi répétitif de cette forme de silence, la suspension, saurait consacrer le procès de la linéarité et confirmer son refus d'écrire une action suivie : un roman. Le langage de la suspension tel qu'il vient

2. Ces interventions sont souvent marquées par l'interpellation du « lecteur».

3. Pour ne citer que cet exemple : «Un faiseur de roman n'y manquerait pas, mais je n'aime pas les romans $[\ldots] »(2013: 255)$. 
dêtre examiné ne saurait marquer la particularité du texte sans les apports rhétoriques des ellipses narratives y mêlées.

\section{Le récit tronqué ou l'ellipse narrative}

En dépit de l'ampleur de la structure romanesque, du caractère bavard des personnages, du goût de l'auteur pour la digression, « déjà réputé digressionniste » (Sabry, 1992: 5) et du langage dans forme orale, «bavard par sa nature (Boilleau, 1999: 112), le roman présente des lacunes au niveau de la narration. L'incipit en témoigne déjà :

Comment s’étaient-ils rencontrés ? Par Hasard, comme tout le monde. Comment s'appelaient-ils ? Que vous importe ? D’où venaient-ils ? Du lieu le plus prochain. Où allaient-ils ? Est-ce que l'on sait où l'on va? (2013:7).

L'auteur y omet d'apporter un éclairage sur ses personnages : ou il en présente des informations vagues ( Par hasard / « Du lieu le plus prochain ») ou il évite - en esquivant - d'en parler (« Que vous importe? » / «Est-ce que l'on sait où l'on va ?»). Le silence sur les circonstances qui ont réuni les deux personnages Jacques et son Maître sur leur identité et sur la destination de leur voyage ne sont pas les seuls retranchements (Boilleau, $1999: 112)^{4}$ dans le roman. Le narrateur évite aussi de préciser les lieux que traversent ses personnages :

[...] ils furent accueillis par un orage qui les contraignit de s'acheminer ... - Où ? Où ? Lecteur, vous êtes d'une curiosité bien incommode [...] Si vous insistez je vous dirai qu'ils s'acheminèrent vers... Oui, pourquoi pas ?... vers [...] (2013:28-29).

Il évite tout de même de dire les raisons de leur déplacement. C'est la conversation avec l'hôtesse qui révèle la nature errante de leur voyage : les deux voyageurs ne savent pas, en fait, "s'ils vont loin ", s'ils "suivent quelqu'un ", s'ils ont " des affaires ", s'ils « voyagent pour leur plaisir » « ou pour leur peine» (2013: 108).

Le narrateur fictif, détenteur de la trame diégétique, troue quelquefois ses discours. Il omet un épisode du récit initial concernant la «troupe d'hommes armés » qui s’avançaient vers Jacques et son maître $(2013: 18)$ lors de leur départ de l'auberge :

Vous allez croire que c’étaient les gens de l'auberge [...]. Vous allez croire que le matin on avait enfoncé leur porte faute de clefs, [...] Jacques le crut [...] Vous allez croire que cette armée tombera sur Jacques et son maître [...] et il ne tiendrait qu’à moi que tout cela n'arrivât, mais adieu la vérité de l'histoire, adieu le récit des amours de Jacques. Nos deux

4. Boilleau souligne que cette manière elliptique de l'écriture est propre à la forme orale de la langue, bien manifeste dans le style de Diderot de Jacques le fataliste. 
voyageurs n'étaient point suivis. J'ignore ce qui se passa dans l'auberge après leur [Jacques et son maître] départ (2013: 19).

Il passe sous silence l'épisode montrant l'hospitalité du "lieutenant » avec les voyageurs éponymes : "Si je ne vous ai pas dit plutôt que Jacques et son maître avaient passé par Conches, et qu'ils avaient logé chez le lieutenant, c'est que cela ne m'est pas revenu plus tôt » $(2013: 35)$. Notons que le retour sur ce passage omis n'est point fortuit. Si le narrateur n'a pas pris compte de l'oubli, le dénouement ne serait pas compris. Car c'est ce lieutenant lui-même qui a sorti le maitre de Jacques, incarcéré pour meurtre, de la prison ${ }^{5}$. Le narrateur omet encore la fin du récit initial : «Et moi, dit-il, je m'arrête, parce que je vous ai dit de ces deux personnages tout ce que j'en sais » $(2013: 303)$. Cette fin sera rapportée par l'éditeur au terme du roman dans le « troisième paragraphe » du «manuscrit » :

Le troisième paragraphe nous montre Jacques, notre pauvre fataliste [...] Jacques obtint sa place [le concierge du château] et épouse Denise [...] aimé de Desglands, chéri de son maître et adoré de sa femme, [...] $(2013: 306-308)$.

Il manque également de s'attarder sur un trait caractéristique de son personnage principal et il n'en parle que tardivement :

J'ai oublié de vous dire, lecteur, que Jacques n'allait jamais sans une gourde pleine du meilleur ; elle était suspendue à l'arçon de sa selle. À chaque fois que son maitre interrompait son récit par quelques questions, il détachait sa gourde, en buvait un coup à la régalade, et ne la remettait à sa place que quand son maître avait cessé de parler. J’avais encore oublié de vous dire que dans les cas qui demandaient de la réflexion, son premier mouvement était d'interroger sa gourde (2013: 239).

Le silence s'installe aussitôt que Jacques achève de raconter les malheurs du vicaire malmené tour à tour par le jeune Jacques et par le mari de Suzon. Le narrateur ne reprend son récit qu’après avoir marqué une lacune par « quelques lignes ponctués »et ce en disant, par le truchement du maître : «Rien n'est plus triste dans ce monde que d’être un sot... » (2013 : 242). L'apophtegme qui clôt une séquence ridiculisant sans scrupule un homme de religion est sans auteur mais il est tentant de l'attribuer au maître qui vient d'annoncer qu'il « n'aime pas les prêtres » $(2013: 235)$. Cette ellipse n'est pas marquée dans le texte du roman mais c'est l'éditeur qui l'y rapporte. Diderot semble s' « amuser ${ }^{6}$ à rassembler son puzzle :

5. «Cependant la maréchaussée qui suivait son maitre à la piste, l'avait atteint, saisi et constitué dans une autre prison. Il en était sorti par les bons offices du commissaire qui l'avait si bien servi dans sa première aventure» $(2013: 307)$.

6. L'expression est de Diderot lui-même; il s'en sert au moment où il se rend compte de ses multiples digressions sur le lieu qui pourrait protéger les deux voyageurs des intempéries : "Vous allez dire que je m’amuse, et de mes deux voyageurs, je me jette dans l'allégorie, [...] ${ }^{\prime}(2013: 29)$. 
Il y a ici une lacune vraiment déplorable dans la conversation de Jacques et de son maître. Quelque jour un descendant de Nodot, du président de Brosses, de Freinshémius, ou du père Brottier, la remplira peut-être, et les descendants de Jacques ou de son maître, propriétaire du manuscrit, en riront beaucoup. [...] Après quelques lignes ponctuées qui annoncent la lacune on lit: « Rien n'es plus triste dans ce monde que d'être un sot ... » Estce Jacques qui profère cet apophtegme ? Est-ce son maître? (2013 : 242).

S'agissant des amours de Jacques, l'auteur en efface l'épisode dans lequel Denise s'offre, en vain, à Jacques. Il éclipse également la scène du baiser. Les deux séquences seront rapportées par "l'éditeur » respectivement dans le " premier » et le "second » paragraphe de son «manuscrit» :

Voici le premier [paragraphe] qui suppose une seconde lacune dans l'entretien de Jacques et de son maître. Un jour de fête que le seigneur du château était à la chasse [...] Jacques était levé. Denise était assise à côté de lui [...]. Ce procédé toucha sensiblement Denise (2013 : 304-305).

Voici le second paragraphe [...] Une autre fois c'était le matin, Denise était venue panser Jacques. [...] Denise s’approcha en tremblant ; arrivée à la porte de Jacques, elle s'arrêta [...] Denise s'offrit à le soulager [...] c'est qu'apparemment il n'en voulait pas encore faire sa femme (2013: 305-306).

En outre le narrateur fait taire Jacques sur la scène d'amour entre l'aubergiste et son époux : "Je ne vous dirais point, dit-il, ce qui se passait entre eux, mais la femme après avoir répété [...]» $(2013: 27)$. Il le fait taire aussi, plus loin, selon les souhaits de son maître " pressé » $(2013: 88)$ de savoir si son valet tombe amoureux de la femme du chirurgien ou de sa fille :

Ah ! Jacques, cela ne se peut. Fais-moi grâce, je te prie, et de la description de la maison, et du caractère du docteur, et de l'humeur de la doctoresse, et des progrès de ta guérison ; saute, saute par-dessus tout cela. Au fait, allons au fait (2013: 88).

Enfin Jacques a interrompu son récit. Il a toujours dit qu'il « ne finirait pas l'histoire [de ses amours]» $(2013$ : 303). Il se tait volontairement sur la conclusion :

Après ces mots il s'arrêta tout court et dit : «Je ne saurais, il m’est impossible d'avancer, il me semble que j'aie derechef la main du destin à la gorge, et que je me la sente serrer ; pour Dieu, monsieur, permettez que je me taise. [...] » $(2013: 302)$.

Le narrateur néglige d'insérer dans les conversations de Jacques et de son maître une réflexion sur les attitudes de Mme de La Pommeraye. Diderot en prend conscience et s'en charge pour combler le vide : 
Et vous croyez, lecteur, que l'apologie de Mme de La Pommeraye est plus difficile à faire ? Il vous aurait peut-être été plus agréable d'entendre là-dessus Jacques et son maître ; mais ils avaient à parler de tant d'autres choses plus intéressantes, qu'ils auraient vraisemblablement négligé celle-ci. Permettez donc que je m’en occupe un moment (2013: 172).

En ce qui concerne les amours du maître de Jacques, le narrateur omet de rapporter le contenu de la lettre d'Agathe tout en justifiant ses choix esthétiques :

Lecteur, vous suspendez ici votre lecture ; qu'est-ce qu'il y a ? Ah ! je crois vous comprendre, vous voudriez voir cette lettre. Mme Riccoboni n'aurait pas manqué de vous la montrer. Et celle que Mme de La Pommeraye dicta aux deux dévotes, je suis sûre que vous l'avez regretté. Quoiqu'elle fût tout autrement difficile à faire que celle d'Agathe, et que je ne présume pas infiniment de mon talent, je crois que je m'en serais tiré, mais elle n'aurait pas été originale ; ç’aurait été comme ces sublimes harangues de Tite-Live dans son Histoire de Rome, ou du cardinal Bentiviglio dans ses Guerres de Flandre. On les lit avec plaisir, mais elles détruisent l'illusion ; un historien, qui suppose à ses personnages des discours qu'ils n'ont pas tenus peut aussi leur supposer des actions qu'ils n'ont pas faites. Je vous supplie donc de vouloir bien vous passer de ces deux lettres et de continuer votre lecture $(2013: 263)$.

Une autre ellipse. Le narrateur se tait un moment, avant de se rattraper, sur la scène qui présente l'hôtesse racontant aux deux voyageurs l'histoire de Mme de La Pommeraye et du comte d'Arcis :

Lecteur, j'avais oublié de vous peindre le site des trois personnages dont il s'agit ici, Jacques, son maître, et l'hôtesse ; faute de cette attention, vous les avez entendu parler, mais vous ne les avez point vus; il vaut mieux tard que jamais. Le maître, à gauche, en bonnet de nuit, en robe de chambre, était étalé nonchalamment dans un grand fauteuil de tapisserie, son mouchoir jeté sur le bras du fauteuil, et sa tabatière à la main. L'hôtesse, sur le fond, en face de la porte, proche de la table, son verre devant elle. Jacques, sans chapeau, à sa droite, les deux coudes appuyés sur la table, et la tête penchée entre deux bouteilles, deux autres étaient à terre à côté de lui... (2013 : 143).

Outre les ellipses narratives au niveau du récit initial, on en repère d'autres au niveau des commentaires de Diderot. Il était parfois des suites possibles au récit qu’il raconte :

Vous allez croire que cette petite armée tombera sur Jacques et son maître, qu'il y aura une action sanglante, des coups de bâton donnés, des coups de pistolets tirés, et il ne teindrait qu'à moi que tout cela n'arrivât, mais adieu la vérité de l'histoire, adieu le récit des amours de Jacques (2013: 19). 
Parfois il tait des arguments à propos d'un sujet, en l'occurrence le fatalisme :

Vous concevez lecteur jusqu'où je pourrais pousser cette conversation sur un sujet dont on a tant parlé, tant écrit depuis deux mille ans, sans en être d'un pas plus avancé. Si vous me savez peu de gré de ce que je vous dis, sachez-m'en beaucoup de ce que je ne vous dis pas $(2013: 13)$.

Le romancier varie sur le thème de l'ellipse narrative. Il retranche volontairement des données et justifie son choix. Il « oublie », pour se rattraper, un épisode, un portrait ou une scène et il suspend la narration pour la reprendre, grâce au «manuscrit ». Ces formes d'ellipses troublent la linéarité du récit sans pour autant le dépareiller. Le romancier semble s'en servir pour briser le totem du récit suivi. Le goût de la discontinuité trouve son pendant dans l'écriture brisée du silence qu'exprime le ponctuant des trois points de suspension.

\section{Les trois points de suspension ou la parole suspendue}

Le texte de Diderot présente une pléthore de points de suspension ${ }^{7}$. Ce signe de ponctuation se lit dans tous les énoncés. Il marque non pas un arrêt significatif de l'achèvement d'une unité de sens tel le point, mais une suspension - volontaire ou involontaire - de la parole. Il fut d'ailleurs désigné par " points de coupure » ou " points poursuivants » (Rault, $2015: 18$ ). Il introduit un vide typographiquement visible dans la phrase en même temps qu'il installe un silence dans la chaîne discursive. Ce silence n'est pourtant pas caduc; ce n'est pas « rien » (Van Den Heuvel, 1985 : 68). Il s'annonce comme une unité du système sémiotique. Il dit autant que la parole articulée, si ce n'est pas plus.

En effet le signe en trois points fait office d'une litote : il dit peu pour dire mieux. Il dit l'éminence de la douleur ainsi que la vivacité du plaisir amoureux : « [...] je m’écriai : «Ah ! le genou... » Et le mari s'écria : «Ah ! ma femme...» (2013: 27). Il communique la drôlerie : « [...] son cheval [...] s'arrête tout court entre des fourches patibulaires... [...]. Voilà une singulière allure de cheval de mener son cavalier au gibet... » $(2013: 65)$ et il dit la pudeur puisqu'il tait des propos pour leur obscénité ou leur incommodité : «Ce n'est pas pour se faire prier, mais c'est que vous êtes quelquefois d'un dur ! ... c'est que... c'est que...» (2013:25). Il transmet la perplexité de Jacques, blessé au genou, face à son mal : «Non, pardieu! monsieur, je ne me moque pas! Il y a là je ne sais combien d'os, de tendons, et bien d'autres choses qu'ils appellent je ne sais comment ...» (2013 : 9). Il laisse voir la colère du maître incapable d'appréhender le fatalisme de son valet : "Que le diable t'emporte avec ton imperti-

7. Nous avons repéré dans l'édition que nous utilisons et qui est composée de 301 pages 591 occurrences. 
nent dicton ! ...» $(2013: 14)$ et l'horreur qui s'empare de lui, coincé par les brigands : "Si... si... si... etc. » (2013 : 15). Le ponctuant dit également le chagrin d'un amant abandonné : « Jacques pleurant à chaudes larmes, lui dit d'un ton dur et amer : "C'est que vous ne m'aimez pas...” » (2013 : 304), la beauté d'une main convoitée : " jolies mains ... Ah ! mon maître, les jolies mains ! ... C’est que ces mains là..., " (2013: 88), la souffrance d'une femme ayant perdu son emploi : "Que je suis malheureuse ! je suis ruinée, je suis ruinée ! ...» (2013: 89); etc. Les trois points expriment aussi le pathétique. La scène des retrouvailles à la fin du roman en témoigne :

Qu'est devenu mon pauvre Jacques... [...] - Et vous comment se fait-il que je vous rencontre ici... [...] C'est vous monsieur Jacques combien vous m'avez fait pleurer !... [...] C'est lui qui nous a sauvé la vie à tous... (2013: 307).

Ils favorisent, étant le signe de l'endophasie, la mise en texte de la voix de l'inconscience : "À ce mot de Nanon, Jacques dit à part lui : "Ah ! c'est sa fille qu'on a maltraitée, on se mettrait en colère à moins..." " (2013 : 100) ou la voix affaiblie par le plaisir amoureux, telle celui de l'hôtesse : «l'or...eil...le » $(2013: 27)$ ou encore la voix troublée par le choc émotionnel que laisse entendre le vicaire qui venait de surprendre les amours de Jacques et Suzanne dans la grange : " Au meu...meu... meurtre ! au feu...feu...feu...au vo...vo...voleur !...» / Ris...ris...ris bien...so...so...sot que tu es... » / « Met...met... mets-moi à te...te...terre» $(2013$ : 235). L'énoncé non vocalisé ou entrecoupé s'annonce comme un moyen de persuasion. Il sert la réception : il aide le lecteur à mieux s'intégrer dans l'univers de l'histoire. Le discours textuel multiplie les exemples qui montrent que le signe des trois points dit l'influence des affects sur la mise en voix et qu'il est bien approprié à l'expression des émotions.

Outre l'expression de l'émotion et des passions ${ }^{8}$ le ponctuant écrit des actes de langages. Il dit, en fait, l'hésitation - feinte - d'un narrateur qui refuse toujours à son lecteur des détails sur les voyageurs :

[...] ils furent accueillis par un orage qui les contraignit de s’acheminer... - Où ? - Où ? [...] Si vous insistez, je vous dirai qu'ils s'acheminèrent vers ... Oui, pourquoi pas... vers un château immense [...] (2013:28-29),

ou l'incertitude : « Pourquoi pas ?... un fou... attendez... c'est un homme malheureux, et par conséquent un homme heureux et un sage " (2013:18) ou encore une injonction : «[...] je veux qu'il se lève, je le veux, je le veux... [...] il faut qu'il se lève,

8. Les trois points, voix du silence et expression de l'ellipse, sont « des éléments du style de la passion » (Aquien et Molinié, 1996 : 146) ; Lamy rapporte, en fait, dans l'article « ellipse », qu' " une passion violente ne permet jamais de dire tout ce que l'on voudrait dire. La langue est trop lente pour suivre la vitesse de ses mouvements » $(1998: 218)$. En outre, Scaliger disait déjà que "l'omission de mot est le signe d'une très grande passion : admiration, amour, haine, colère » (cité dans Surgers, $2007: 165$ ). 
je le veux, je le veux...» $(2013: 277)$. Il rend au fur et à mesure des échanges la tension entre les interlocuteurs ou leur complicité : l'un reprend ou complète les propos de l'autre aussitôt qu'il est interrompu : «- Et moi, en t'attendant.../ - En m'attendant il était écrit là-haut que vous vous endormiriez, et qu'on vous volerait votre cheval » (2013 : 39)/ « Mon cher maitre.../ - Ah ! la parole t'es enfin revenue» (2013:65)/ " - Mon maître, un prophète ne dirait pas mieux ; mais heureusement .../ - Vous n'y croyez pas trop » (2013:83)/ «- Je ne saurais te le dissimuler, mais pour écarter cette triste idée, ne pourrais-tu pas ?.../ - Reprendre les histoires de mes amours ?... » (2013: 85)/ « Ces chiens, cela est si bon, cela vaut mieux.../ - Que pères, mère, frères, sœurs, enfants, valets, époux...» $(2013: 115)$.

Parallèlement à la fonction pragmatique les trois points par leur matérialité graphique permettent de transgresser le langage. Ils font «apparaître [en fait] que quelque chose est susceptible d'apparaitre $»^{9}$ (Rault, $2015: 14$ ). Par exemple une explication : «Et il avait raison... » (2013: 7), une histoire : «À tout hasard, commence toujours... » (2013: 8), un épisode d'une histoire déjà commencée : « Un jour de fête que le seigneur du château était à la chasse... » (2013 : 302), une suite de plaintes : « [...] le voilà criant à tue-tête : je suis mort ! j’ai le genou cassé !...» (2013:23), le récit d'une altercation : "Là j’entends un vacarme... » (1013:96), une insinuation : "C'était un homme de plaisir, très aimable, croyant peu à la vertu des femmes... » $(2013: 117)$, etc. Le «manque graphique » (Van Den Heuvel, 1985 : 73) s'avère un signe révélateur d'un lieu de tous les possibles. Il sert la lecture participative et aiguise l'interprétation. Il dit « le refus des savoir clos » (Rousseau, 1997 : 47).

De même, à part le fait que les trois points autorisent l'éclatement du « discours textuel» (Van Den Heuvel, 1985 : 99) ils favorisent également la rétention des répétitions et des redites. Ils renvoient effectivement à un propos ou à un récit déjà connu du lecteur et de son interlocuteur. Introduits dans le texte, ils permettent d'éviter tout en l'insinuant de reprendre un épisode du récit initial : " - N'êtes-vous pas le citoyen officieux qui m'a secouru, qui m’a saigné et qui m’a pansé, lorsque mon cheval... » (2013 : 81), de réitérer le slogan de Jacques : « Il fallait que cela fût, cela était écrit là-haut... » (2013 : 303), de revenir sur une triste histoire : «Laissez-moi, je n’ai pas envie de rire. Un pauvre malheureux qui se meurt à notre porte... » $(2013: 25)$, de faire revivre un mal : "Mais pour revenir à une peine que nous connaissons tous deux, l'histoire de mon genou qui est devenu le vôtre par votre chute... » $(2013: 24)$, etc. Le point de suspension s'avère " une marque explicite d'implicite " qui indique "la présence d'un ajout absent », un "non -dit explicite » (Le Bozec Y., Barbet C., De Saussure L. cités dans Rault, 2015 : 46).

Les occurrences des points de suspension dans Jacques le fataliste exemplifient la polyvalence et la polysémie du ponctuant. Celui-ci se révèle, en fait, la traduction langagière des différents états d'âme. Il dit à la fois l'indicible et le tout dire. Rault explique en fait sa «valeur de latence [...] qui pourrait désigner ce qui n'est pas ver-

9. L'italique est de l'auteur. 
balisé $»^{10}$ (2015 : 51). Sa profusion dans le texte renseignerait sur l'ampleur du fonds esthétique et philosophique que Diderot cherche à porter dans son roman car « en tout point, le signe excède » (Rault, 2015 : 55). L’engouement pour ce signe de ponctuation dirait la volonté de l'auteur de « tout dire » tout en déjouant et ridiculisant les conventions du genre qu'il ne cesse de décrier ${ }^{11}$.

\section{Conclusion}

Le silence dans le roman de Jacques le fataliste s'annonce audible voire "vocalisé » (Durrer, 1999: 13). Les récits se taisent du fait des interruptions et cèdent la place aux commentaires, aux digressions, aux réflexions, aux anecdotes, etc. Paradoxalement les pauses génèrent, varient et multiplient le dire. Les ellipses narratives se diversifient. Elles manquent de dire mais elles ne nuisent point à l'unité du récit ${ }^{12}$ puisqu'elles sont souvent complétées grâce au secours du "manuscrit » et à la mémoire d'un auteur qui, pourtant, " oublie ». Elles disent ainsi un silence provisoire, feint. Quant aux points de suspension ils continuent de parler au-delà de leur forme qui rappelle injustement le point, titre de l'achèvement. Par sa nature à caractère latent, le signe de ponctuation dit ce qui est caché, mystérieux et secret. "C’est, [véritablement], le signe qui indique l'affranchissement (libertinus) [...] qui permet de dire sans dire et de dire au-delà du dit ${ }^{13}$ (Rault, $2015: 15$ ).

Le silence dans le roman est loin d'être l'expression d'un vide narratif (les ellipses) ou graphique (les points de suspension); c'est plutôt la concentration d'une fécondité discursive. Les différentes formes du silence qui brisent régulièrement la continuité de la narration semblent subvertir les canons en usage de l'écriture romanesque. L'esthétique de la fraction et de la rupture - effacement, atermoiement ou latence - qui installe ce que K.-Toumarkine appelle l'« ordre du discontinu » (Diderot, $2012: 23$ ) s'investit de trois fonctions : d'abord elle justifie le classement de Jacques le fataliste sur le paradigme de l'" antiroman" (Sermain, 2011 : 204) que la critique moderne lui attribue, ensuite elle rattache par le biais de l'" écriture rhapsodique » (Soler, 2001 : 341) le roman au genre picaresque qui semble inspirer Diderot et dans lequel le motif du voyage permet des variétés illimitées de rencontres et de récits et enfin elle lui assigne la particularité d'un texte qui anticipe, par le truchement de cette sorte de fragmentation, le roman moderne.

10. L'italique est de l'auteur.

11. Dans son ouvrage Le dilemme du roman au XVIII siècle George May (1963) s'attarde sur les raisons pour lesquelles la critique littéraire du siècle s'érige contre le roman de l'époque.

12. Ceci correspond à la définition de l'ellipse, au niveau microstructurel, chez Fontanier : « L'ellipse consiste dans la suppression de mots qui seraient nécessaires à la plénitude de la construction, mais que ceux qui sont exprimés font assez entendre pour qu'il ne reste ni obscurité ni incertitude » (1968:305).

13. L'italique est de l'auteur. 


\section{BIBLIOGRAPHIE}

Aquien M., Molinié G. 1996. Dictionnaire de Rhétorique et de Poétique. Paris. La Pochothèque. Le livre de Poche.

Boilleau A.-M. 1999. Liaison et liaisons dans les lettres de Diderot à Sophie Volland. Paris. Honoré Champion.

Coulet. H. 1967. Le roman jusqu’à la Révolution. Paris. Armand Colin.

Diderot D. [1997] 2012. Jacques le fataliste et son maître. Paris. Flammarion.

Diderot D. 2013. Jacques le fataliste et son maître. Paris. Hatier.

Durrer S. 1999. Le dialogue dans le roman. Paris. Armand Colin.

Lamy B. 1998. La rhétorique ou l'art de parler. Paris. Honoré Champion.

May G. 1963. Le dilemme du roman au XVIII siècle. Paris. PUF.

Rault J. 2015. Poétique du point de suspension. Essai sur le signe du latent. Nantes. Éditions Nouvelles Cécile Defaut.

Rousseau N. 1997. Diderot : l’écriture romanesque à l’épreuve du sensible. Paris. Honoré Champion.

Sabri R. 1992. Stratégies discursives. Paris. Éditions de l'École des Hautes Études en Sciences Sociales.

Soler P. 2001. Genres, formes, tons. Paris. PUF.

Sermain J.-P. 2011. Le roman jusqu’à la Révolution française. Paris. PUF.

Surgers A. 2007. Et que dit ce silence? : la rhétorique du visible. Paris. Presses Sorbonne Nouvelle.

Van Den Heuvel P. 1985. Parole. Mot. Silence. Pour une poétique de l’énonciation. Paris. Librairie José Corti.

\section{The aspects and the stakes of the silence in Jacques le fataliste of Diderot}

ABSTRACT: The novel presents a variety of writings of silence; but we limit ourselves, in the context of the present work, to the most relevant aspects, namely interruptions in narratives, narrative ellipses and point of suspension as the particular language of silence. These three modalities nevertheless serve aprolix discourse, and are innovative. Suddenly the novel exemplifies what contemporary literature calls the anti-novel.

Keywords: silence, points of suspension, narrative ellipse, interruption, story. 